\title{
Controlling the Tax Evasion Dynamics via Majority-Vote Model on Various Topologies"
}

\author{
Francisco W. S. Lima \\ Dietrich Stauffer Computational Physics Lab, Departamento de Física, Universidade Federal do Piauí, Teresina, Brazil \\ Email: fwslima@gmail.com
}

Received October 27, 2011; revised November 18, 2011; accepted November 25, 2011

\begin{abstract}
Within the context of agent-based Monte-Carlo simulations, we study the well-known majority-vote model (MVM) with noise applied to tax evasion on simple square lattices (LS), Honisch-Stauffer (SH), directed and undirected Barabasi-Albert (BAD, BAU) networks. In to control the fluctuations for tax evasion in the economics model proposed by Zaklan, MVM is applied in the neighborhood of the noise critical qc to evolve the Zaklan model. The Zaklan model had been studied recently using the equilibrium Ising model. Here we show that the Zaklan model is robust because this can be studied using equilibrium dynamics of Ising model also through the nonequilibrium MVM and on various topologies cited above giving the same behavior regardless of dynamic or topology used here.
\end{abstract}

Keywords: Opinion Dynamics; Sociophysics; Majority Vote; Nonequilibrium

\section{Introduction}

The Ising model $[1,2]$ has become a excellent tool for to study other models of social application. The Ising model was already applied decades ago to explain how a school of fish aligns into one direction for swimming [3] or how workers decide whether or not to go on strike [4]. In the Latané model of Social Impact [5] the Ising model has been used to give a consensus, a fragmentation into many different opinions, or a leadership effect when a few people change the opinion of lots of others. To some extent the voter model of Liggett [6] is an Ising-type model: opinions follow the majority of the neighbourhood, similar to Schelling [7], all these cited models and others can be found out in [8]. Already Föllmer (1974) [9] applied the Ising model to economics. Realistic models of tax evasion appear to be necessary because tax evasion remain to be a major predicament facing governments [10-13]. Experimental evidence provided by Gächter [14] indeed suggests that tax payers tend to condition their decision regarding whether to pay taxes or not on the tax evasion decision of the members of their group. Frey and Torgler [15] also provide empirical evidence on the relevance of conditional cooperation for tax morale. Following the same context, recently, Zaklan et al. [16] developed an economics model to study the problem of tax evasion dynamics using the Ising model through MonteCarlo simulations with the Glauber and heatbath algorithms (that obey detailed-balance equilibrium) to study

\footnotetext{
*This paper is dedicated to Dietrich Stauffer.
}

the proposed model. I have introduced for the first time the use of local majority rules in social systems. I also include a review paper on all my contributions to the field of sociophysics. Another one shows that a unifying paper on all discrete opinion models. I hope you will find these papers of interest.

Grinstein et al. [17] have argued that nonequilibrium stochastic spin systems on regular square lattices with up-down symmetry fall into the universality class of the equilibrium Ising model [18]. This conjecture was confirmed for various Archimedean lattices and in several models that do not obey detailed balance [19-22]. The majority-vote model (MVM) is a nonequilibrium model proposed by M. J. Oliveira in 1992 [20] and defined by stochastic dynamics with local rules and with up-down symmetry on a regular lattice shows a second-order phase transition with critical exponents $\beta, \gamma$, and $v$ which characterize the system in the vicinity of the phase transition identical with those of the equilibrim Ising model [1] for regular lattices. Lima et al. [23] studied MVM on VD random lattices with periodic boundary conditions. These lattices posses natural quenched disorder in their connections. They showed that presence of quenched connectivity disorder is enough to alter the exponents and from the pure model and therefore that is a relevant term to such non-equilibrium phase-transition with disagree with the arguments of Grinstein et al. [17].

Recently, simulations on both undirected and directed scale-free networks [24-30], random graphs [31] and social networks [32-35], have attracted interest of re- 
searchers from various areas. These complex networks have been studied extensively by Lima et al. in the context of magnetism (MVM, Ising, and Potts model) [3539], econophysics models [16,40] and sociophysics model [41]. In the present work, we study the behavior of the tax evasion on two-dimensional LS, BAD and BAU networks, and SH networks using the dynamics of MVM, furthermore add a policy makers's tax enforcement mechanism consisting of two components: a probability of an audit each person is subject to in everyperiod and a length of time detected tax evaders remain honest. We aim here is to extend the study of Zaklan et al. [16], which illustrates how different levels of enforcement affect the tax evasion over time, to dynamics of MVM as an alternative model of nonequilibrium to the Ising model that is capable of reproduce the same results for analysis and control of the tax evasion fluctuations. Then, we show that the Zaklan model is very robust for equilibruim and nonequilibrium models and also for various topologies used here. We show that the choice of using the Ising (equilibrium dynamics) or MVM (nonequilibrium dynamics) used to evolve the Zaklan model is irrelevant, because the results obtained in this work are about the same for both Ising and MVM. The Zaklan model also is robust, because it works on LS, SH network, BAD and BAU networks. We show that for different topologies the Zaklan model reaches our objective, that is, to control the tax evasion of a country (Germany and others). This does not occur with other models as Axelrod-Ross model for evolution of ethnocentrism [41], because the results are different depending of the topology of the network. The Ising model also is not robust, because on directed BA network occur with other models as Axelrod-this no phase transition present as also on directed LS, 3D, 4D and directed hypercubics lattices [42]. As described above, the MVM was proposed by M.J. Oliveira in 1992 [22] in order to improve the criterion of Grinstein et al. [17], initially described above. In the order to achieve his goal he used 44 (LS) Archimedean lattice. However, also with the aim of improve this criterion other researchers studied MVM on several other topologies that are not Archimedeans [39,43-48]. To their surprise all results obtained for the critical exponents are different from results obtained by M. J. Oliveira, and are also different for each topology used. Pereira et al. [49] then concluded that MVM has different universality classes which depend only on the topology used, and that all have one thing in common that is their effective dimension, obtained by critical exponents for each topology used, equals Deff $=1$. Here, we show that the Zaklan model behavior is identical for all topologies or dynamics studied here. Therefore, we believe that this model is very robust, different the other models cited above. Galam [50-53] introduced for the first time local majority rules in social systems to the field of sociophysics using discrete opinion models. Here, we also hope to introduce for the first time the use of MVM to the field of sociophysics or econophysics using discrete opinions as in the Zaklan. Therefore, we do not live in a social equilibrium, any rumor or gossip can lead to a government or market chaos and we believe that nothing is better than a nonequilibrium model (MVM) to explain events of nonequilibrium. Stock market generalized to market, in order to include currency exchange. The remainder of our paper is organised as follows. In Section 2, we present the Zaklan model evolving with dynamics of MVM. In Section 3 we make an analysis of tax evasion dynamics with the Zaklan model on two-dimensional square lattices using MVM for their temporal evolution under different enforcement regimes; we discuss the results obtained. In Section 4 we show that MVM also is capable to control the different levels of the tax evasion analysed in Section 3, as it was made by Zaklan et al. [16] using Ising models. We use the enforcement mechanism cited above on various structures: SL, SH network, BAD and BAU network; we discuss the resulting tax evasion dynamics. Finally in Section 5 we present our conclusions about the study of the Zaklan model using MVM.

\section{Zaklan Model}

On a square lattice each site of the lattice is inhabited, at each time step, by an agent with "voters" or spin variables $\sigma$ taking the values +1 representing an honest tax payer, or -1 trying to at least partially escape her tax duty. Here is assumed that initially everybody is honest. Each period individuals can rethink their behavior and have the opportunity to become the opposite type of agent they were in previous period. In each time period the system evolves by a single spin-flip dynamics with a probability given by

$$
w_{i}(\sigma)=\frac{1}{2}\left[1-(1-2 q) \sigma_{i} S\left(\sum_{\delta=1}^{k_{i}} \sigma_{i+\delta}\right)\right]
$$

where $S(x)$ is the sign \pm 1 of $x$ if $x \neq 0 . S(x)=0$ if $x=0$, and the summation runs over all $k_{i}$ nearestneighbour sites $\sigma_{i+\delta}$ of $\sigma_{i}$. In this model an agent assumes the value \pm 1 depending on the opinion of the majority of its neighbors. The control noise parameter $q$ plays the role of the temperature in equilibrium systems and measures the probability of aligning antiparallel to the majority of neighbors. Then various degrees of homogeneity regarding either position are possible. An extremely homogenous group is entirely made of honest people or tax evaders, depending the sign $S(x)$ of the majority of neighbhors. If $S(x)$ of the neighbors is zero the agent $\sigma_{i}$ will be honest or evader in the next 
time period with probability $1 / 2$. We further introduce a probability of an efficient audit $(p)$. Therefore, if tax evasion is detected, the agent must remain honest for a number $k$ of time steps. Here, one time step is one sweep through the entire lattice.

\section{Controlling the Tax Evasion Dynamics}

Here, we first will present the baseline case, i.e., no use of enforcement, for different network structure. We use for LS, BAD and BAU network, and SH network. All simulation are performed over 25,000 time steps, as shown in Figure 1. For very low noises the part of autonomous decisions almost completely disappears. The individuals then base their decision solely on what most of their neighbours do. A rising noise has the opposite effect. Individuals then decide more autonomously. For MVM it is known that for $q>q_{c}$, half of the people are honest and other half cheat, while for $q>q_{c}$ states dominated by cheating or by correlated changed into dominated; you always have correlations compliance prevail for most of the time. Because this behavior we set some values close to $q_{c}$, where the case that agents distribute in equal proportions onto the two alternatives is excluded. Then having set the noise parameter, $q$, close to $\left(q_{c}=0.075\right)$ on the square lattice, as suggested in Section 3, we vary the degrees of punishment $(k=1,10$ and $50)$ and audit probability rate $(p=0.5 \%, 10 \%$ and $90 \%)$. Therefore, if tax evasion is detected, the enforcement mechanism $(p)$ and the period time of punishment

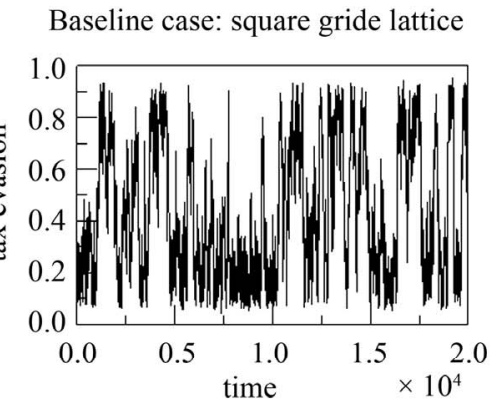

(a)

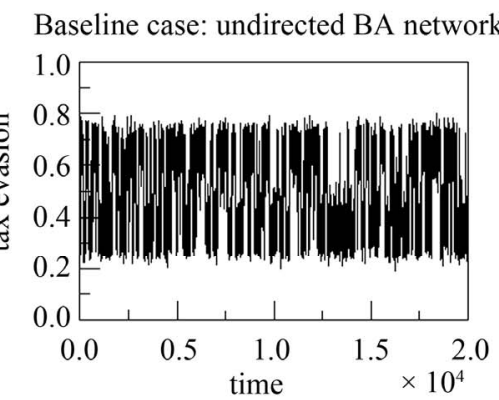

(c) $k$ are triggered in order of to control the tax evasion level. The individual remain honests for a certain number of periods, as explained before in Sections 2 and 3. We also extend our study to other networks as the SH network, BAD and and BAU networks with $\mathrm{N}=400$ sites. As before the initial configurations is with all honest agents $\left(\sigma_{i}\right)$ at fixed "Social Temperature" $(q)$. Here, we have been performed simulations of 25,000 time steps.

In Figure 1 we plot the baseline case $k=0$, i.e., no use of enforcement, for the LS (a), SH (b), BAU (c), and BAD (d) for dynamics of the tax evasion over 25,000 time steps. Although everybody is honest initially, it is impossible to predict which level of tax compliance will be reached at some time step in the future.

Figure 2 illustrates different simulation settings for square lattice, for each considered combination of degree of punishment $(k=1,10$ and 50$)$ and audit probability rate $(p=0.5 \%, 10 \%$ and $90 \%)$, where the tax evasion is plotted over 20,000 time steps. Here we show that even a very small level the enforcement ( $p=0.5 \%$ and $k=1$ ) suffices to reduce fluctuations in tax evasion and to establish mainly compliance. Both a rise in audit probability (greater $p$ ) and higher penalty (greater $k$ ) work to flatten the time series of tax evasion and to shift the band of possible non-compliance values towards more comp liance. However, the simulations show that even extreme enforcement measures ( $p=90 \%$ and $k=50)$ cannot fully solve the problem of tax evasion.

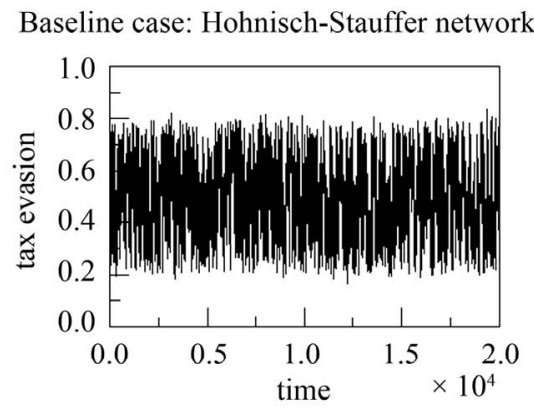

(b)

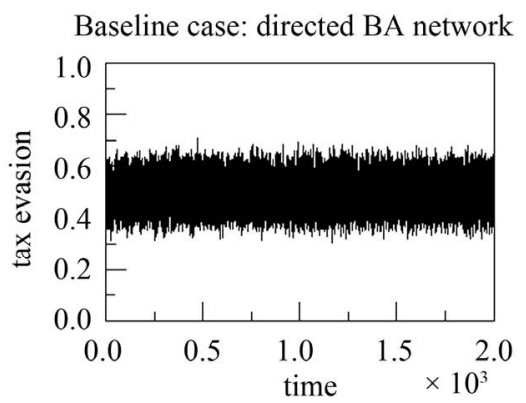

(d)

Figure 1. Baseline case for different network structure. Where we use $\boldsymbol{q}=\mathbf{0 . 9 5} \boldsymbol{q}_{\mathrm{c}}$ on different networks. All simulation are performed over 25,000 time steps. 
In Figure 3 we display tax evasion for BAD and BAU networks, SH networks for different enforcement for $k=$ 1,10 , and 50 with the same audit probability $p=1 \%$. We observe for BAD ou BAU network that the tax evasion level decreases with increasing time periods $k$ of punishment, similar behavior also occurs for SH network.

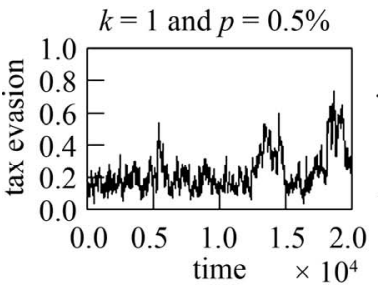

(a)

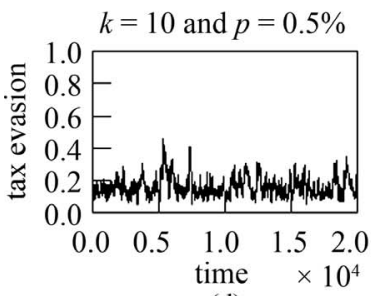

(d)

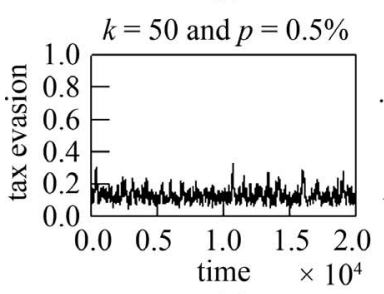

(g)

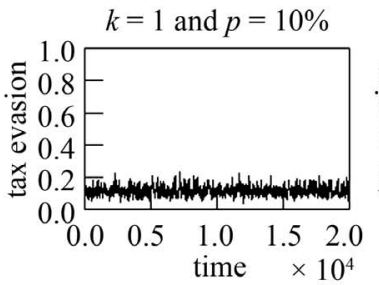

(b)

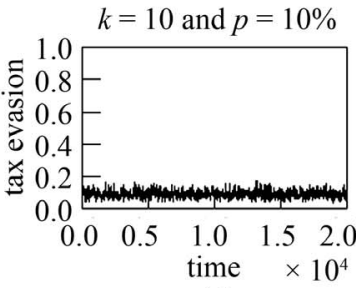

(e)

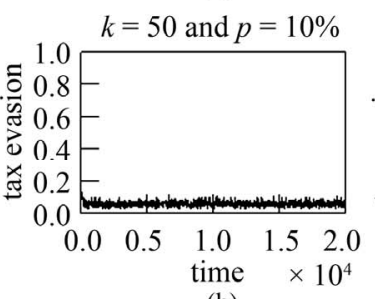

(h)

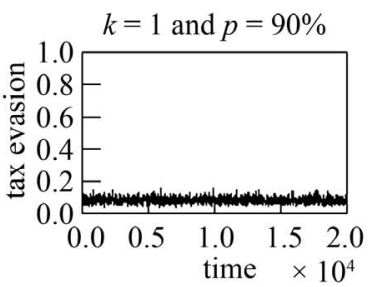

(c)

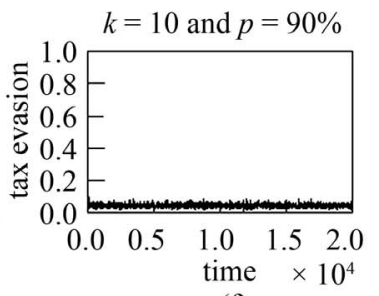

(f)

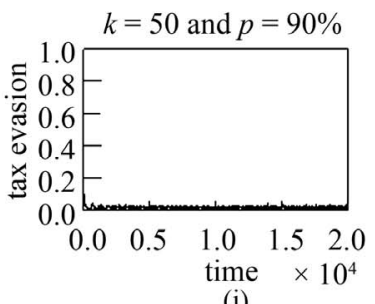

(i)

Figure 2. The square lattice model of tax evasion with various degrees of enforcement $q=0.95 q_{c}$ and 20,000 time steps.

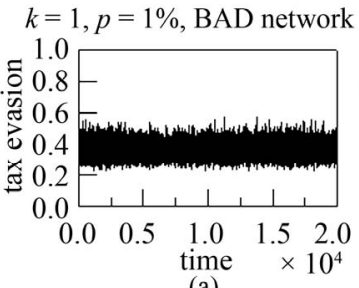

(a)

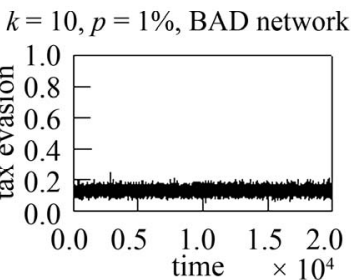

(d)

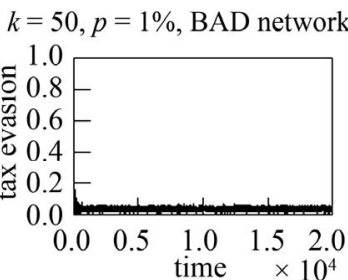

(g)

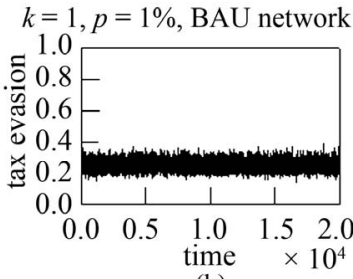

(b)

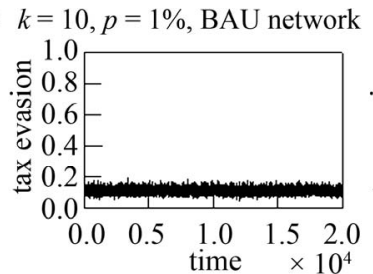

(e)

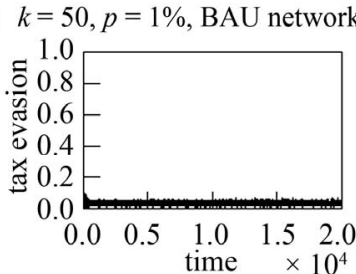

(h)

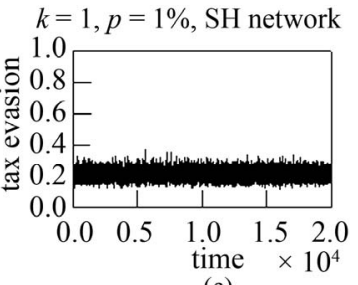

(c)

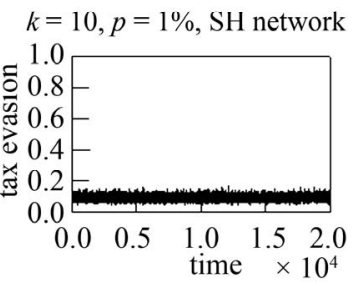

(f)

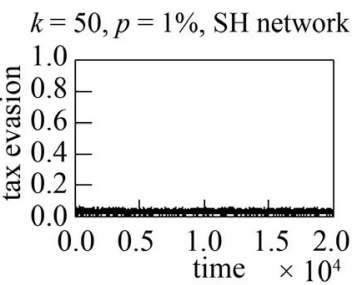

(i)

Figure 3. Display tax evasion for different enforcement regimes for BA and SH Network and for degrees of punishment $k=1$, 10,50 and audit probability rate $p_{a}=4.5 \%$. 


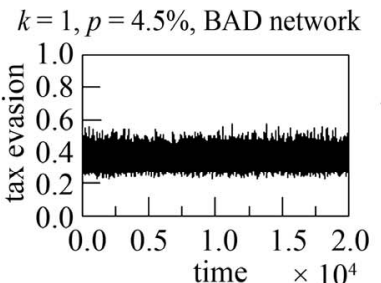

(a)

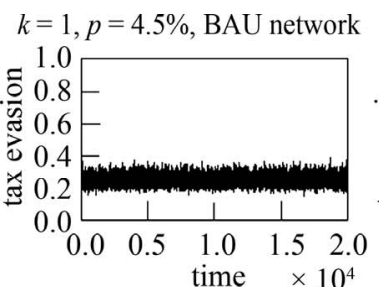

(d)

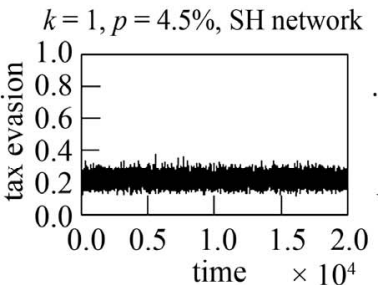

(g)

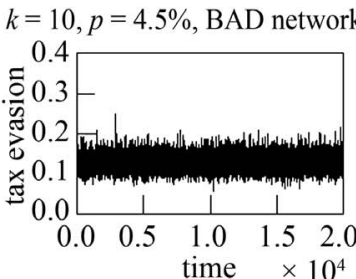

(b)

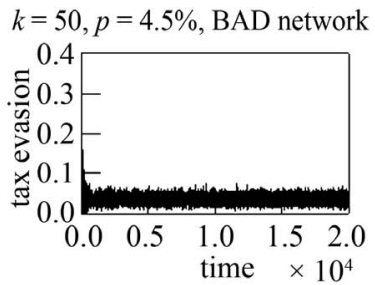

(c)

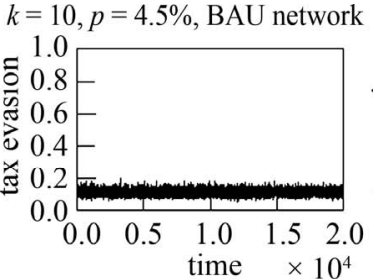

(e)

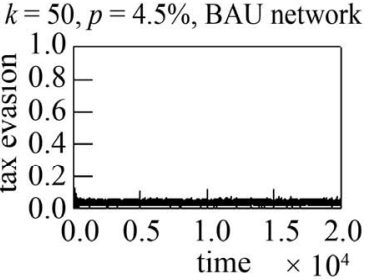

(f)

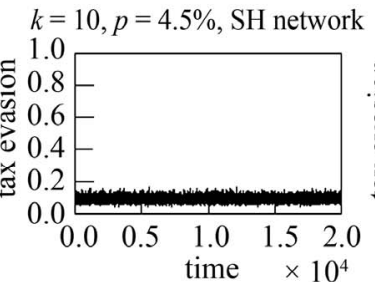

(h)

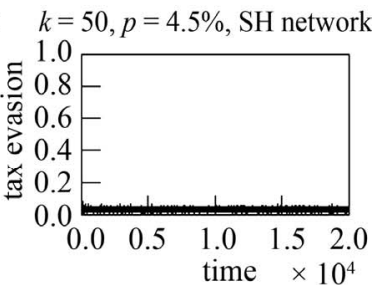

(i)

Figure 4. Display of the tax evasion for different enforcement regimes for BA and SH network. Again, we use 25,000 time steps.

In Figure 4 we plot tax evasion for BAD and BAU networks, and $\mathrm{SH}$ network, again for different enforcement $k=1,10$, and 50, but now with audit probability $p=4.5 \%$. For BAD and BAU, and SH networks the tax evasion level decreases with increasing audit probability $p$ showing that an increase of the audit probability favors the control of tax evasion. In all case studied here, we observed that the time period $k$ of punishment is important to control tax evasion.

\section{Conclusion}

In summary, tax evasion can vary widely across nations, reaching extremely high values in some developing countries. Wintrobe and Gërxhani [54] explains the observed higher level of tax evasion in generally less developed countries with a lower amount of trust that people have in governmen tal institutions. To study this problem Zaklan et al. [16] proposed a model, called here call the Zaklan model, using Monte Carlo simula- tions and a equilibrium dynamics (Ising model) on square lattices. Their results are good agreement with analytical and experimental results obtained by [9-15,54]. In this work we show that the Zaklan model is very robust for analysis and control of tax evasion, because we use a nonequilibrium dynamics (MVM) to simulate the Zaklan model, that is the opposite of the study done by [16] equilibrium dynamics (Ising model), and also on various topologies used here. Our results are qualitatively and quantitatively identical the results obtained by Zaklan et al. [16] giving the same behavior regardless of dynamic or topology. Here, we also hope to have introduced for the first time the use of MVM to the field of sociophysics and econophysics using discrete opinion model as Zaklan model. As we do not live in a social equilibrium and any rumor or gossip can lead to a government or market chaos, we believe that nothing is better than a nonequilibrium model (MVM) to explain events of nonequilibrium. Therefore, as the Zaklan model is a sociophysics and econophysics model, we also believe that the best topology used for simulations of this model are social networks of BAD and SH type.

\section{Acknowledgements}

The author thanks D. Stauffer for many suggestion and fruitful discussions during the development this work and also for the reading this paper. We also acknowledge the Brazilian agency FAPEPI (Teresina-Piauí-Brasil) for its financial support. This work also was supported the system SGI Altix 1350 the computational park CENA-PAD. UNICAMP-USP, SP-BRAZIL.

\section{REFERENCES}

[1] L. Onsager, "Crystal Statistics. I. A Two-Dimensional 
Model with an Order-Disorder Transition," Physical Review, Vol. 65, No. 3-4, 1944, pp. 117-149. doi:10.1103/PhysRev.65.117

[2] R. J. Baxter, "Exactly Solved Models in Statistical Mechanics," Academic Press, London, 1982.

[3] E. Callen and D. Shapero, "A Theory of Social Imitation," Physics Today, Vol. 27, No. 7, 1974, 23 Pages. doi:10.1063/1.3128690

[4] S. Galam, Y. Gefen and Y. Shapir, "Sociophysics: A Mean Behavior Model for the Process of Strike," Journal of Mathematical Sociology, Vol. 9, 1982. doi:10.1080/0022250X.1982.9989929

[5] B. Latané, "The Psychology of Social Impact," American Psychologist, Vol. 36, No. 4, 1981, pp. 343-356.

[6] T. M. Liggett, "Interacting Particles Systems," Springer, New York, 1985. doi:10.1007/978-1-4613-8542-4

[7] T. C. Schelling, "Dynamic Models of Segregation," Journal of Mathematical Sociology, Vol. 1, No. 2, 1971, pp. 143-186. doi:10.1080/0022250X.1971.9989794

[8] D. Stauffer, S. Moss de Oliveira, P. M. C. de Oliveira and J. S. Sá Martins, "Biology, Sociology, Geology by Computational Physicists," Elsevier, Amsterdam, 2006.

[9] H. Föllmer, "Random Economies with Many Interacting Agents," Journal of Mathematical Economics, Vol. 1, 1974, p. 51. doi:10.1016/0304-4068(74)90035-4

[10] K. Bloomquist, "A Comparison of Agent-Based Models of Income Tax Evasion," Social Science Computer Review, Vol. 24, No. 4, 2006, pp. 411-425. doi: $10.1177 / 0894439306287021$

[11] J. Andreoni, B. Erard and J. Feinstein, "Tax Compliance," Journal of Economic Literature, Vol. 36, No. 2, 1998, pp. 818-860.

[12] L. Lederman, "Interplay between Norms and Enforcement in Tax Compliance," Public Law Research Paper No. 50, 2003.

[13] J. Slemrod, "Cheating Ourselves: The Economics of Tax Evasion," Journal of Economic Perspective, Vol. 21, No. 1, 2007, pp. 25-48. doi:10.1257/jep.21.1.25

[14] S. Gächter, "Moral Judgments in Social Dilemmas: How Bad is Free Riding?" Discussion Papers 2006-03 CeDEx, University of Nottingham, 2006.

[15] B. S. Frey and B. Togler, "Managing Motivation, Organization and Governance," IEW-Working Papers 286, Institute for Empirical Research in Economics, University of Zurich, 2006.

[16] G. Zaklan, F. W. S. Lima and F. Westerhoff, "Controlling Tax Evasion Fluctuations," Physica A, Vol. 387, No. 23, 2008, pp. 5857-5861. doi:10.1016/j.physa.2008.06.036

[17] G. Grinstein, C. Jayaprakash and Y. He, "Statistical Mechanics of Probabilistic Cellular Automat," Physical Review Letters, Vol. 55, No. 23, 1985, pp. 2527-2530. doi:10.1103/PhysRevLett.55.2527

[18] J. S. Wang and J. L. Lebowitz, "Phase Transitions and Universality in Nonequilibrium Steady States of Stochastic Ising Models," Journal of Statistical Physics, Vol. 51, No. 5-6, 1988, pp. 893-906. doi:10.1007/BF01014891
[19] M. C. Marques, "Nonequilibrium Ising Model with Competing Dynamics: A MFRG Approach," Physics Letters A, Vol. 145, No. 6-7, 1990, pp. 379-382. doi:10.1016/0375-9601(90)90954-M

[20] M. J. Oliveira, "Isotropic Majority-Vote Model on a Square Lattice," Journal of Statistical Physics, Vol. 66, No. 1, 1992, pp. 273-281. doi:10.1007/BF01060069

[21] F. W. S. Lima, "Majority-Vote Model on (3, 4, 6, 4) and $\left(3^{4}, 6\right)$ Archimedean Lattices," International Journal of Modern Physics C (IJMPC), Vol. 17, No. 9, 2006, pp. 1273-1283. doi:10.1142/S0129183106009849

[22] M. A. Santos and S. J. Teixeira, "Universality Classes in Nonequilibrium Lattice Systems," Statistical Physics, Vol. 78, 1995, p. 963. doi:10.1007/BF02183696

[23] F. W. S. Lima, U. L. Fulco and R. N. Costa Filho, "Majority-Vote Model on a Random Lattice," Physical Review E, Vol. 71, No. 3, 2005, Article ID 036105. doi:10.1103/PhysRevE.71.036105

[24] M. E. J. Newman, S. H. Strogatz and D. J. Watts, "Random Graphs with Arbitrary Degree Distributions and Their Applications," Physical Review E, Vol. 64, No. 2, 2001, Article ID 026118. doi:10.1103/PhysRevE.64.026118

[25] A. D. Sanchez, J. M. Lopez and M. A. Rodriguez, "Nonequilibrium Phase Transitions in Directed Small-World Networks," Physical Review Letters, Vol. 88, No. 4, 2002, Article ID 048701. doi:10.1103/PhysRevLett.88.048701

[26] G. Palla, I. J. Farkas, P. Pollner, I. Derenyi and T. Vicsek, "Directed Network Modules," New Journal of Physics, Vol. 9, No. 6, 2007, pp. 186-207. doi:10.1088/1367-2630/9/6/186

[27] A.-L. Barabási and R. Albert, "Emergence of Scaling in Random Networks," Science, Vol. 286, No. 5439, 1999, pp. 509-512. doi:10.1126/science.286.5439.509

[28] A. Aleksiejuk, J. A. Holyst and D. Stauffer, "Ferromagnetic Phase Transition in Barabasi-Albert Networks," Physica A, Vol. 310, No. 1-2, 2002, pp. 260-266. doi:10.1016/S0378-4371(02)00740-9

[29] M. A. Sumour and M. M. Shabat, "Monte Carlo Simulation of Ising Model on Directed Barabasi-Albert Network," International Journal of Modern Physics C, Vol. 16, No. 4, 2005, pp. 585-589. doi:10.1142/S0129183105007352

[30] M. A. Sumour and M. M. Shabat and D. Stauffer, "Absence of Ferromagnetism in Ising Model on Directed Barabasi-Albert Network," Islamic University Journal (Gaza), Vol. 14, 2006, p. 209.

[31] P. Erd"os and A. Rényi, "Graphy Theory and Probability," Canadian Journal of Mathematics, Vol. 6, 1959, p. 290.

[32] D. J. Watts and S. H. Strogatz, "Collective Dynamics of Small-World Networks," Nature, Vol. 393, 6684, 1998, pp. 440-442. doi:10.1038/30918

[33] S. Wasseman, K. Faust and B. Balobás, "Social Networks Analysis," Cambridge University Press, Cambridge, 1994.

[34] D. Stauffer, M. Hohnisch and S. Pittnauer, "Consensus 
Formation on Coevolving Networks: Groups' Formation and Structure," Physica A, Vol. 370, No. 2, 2006, pp. 734-740. doi:10.1016/j.physa.2006.05.033

[35] F. W. S. Lima, "Majority-Vote on Directed BarabasiAlbert Networks," International Journal of Modern Physics C, Vol. 17, No. 9, 2006, pp. 1257-1265.

[36] F. W. S. Lima, "Ising Model Spin $\mathrm{S}=1$ on Directed Barabási-Albert Networks," International Journal of Modern Physics C, Vol. 17, 2006, p. 257.

[37] F. W. S. Lima, "Potts Model with q States on Directed Barabasi-Albert Networks," Communications in Computational Physics, Vol. 2, 2007, pp. 522-529.

[38] F. W. S. Lima, "Simulation of Majority Rule Disturbed by Power-Law Noise on Directed and Undirected Barabási Albert Networks," International Journal of Modern Physics C, Vol. 19, 2008, p. 1063. doi:10.1142/S0129183108012686

[39] F. P. Fernandes and F. W. S. Lima, " Persistence in the Zero-Temperature Dynamics of the Q-states Potts Model on Undirected-Directed Barabási-Albert Networks and Erdös-Rènyi Random Graphs," International Journal of Modern Physics C, Vol. 19, No. 12, 2008, pp. 1777-1785. doi:10.1142/S0129183108013345

[40] F. W. S. Lima and G. Zaklan, "A Multi-Agent-Based Approach to Tax Morale," International Journal of Modern Physics C, Vol. 19, 2008, pp. 1797-1822. doi:10.1142/S0129183108013357

[41] F. W. S. Lima, T. Hadzibeganovic and D. Stauffer, "Evolution of Ethnocentrism on Undirected and Directed Barabási Albert Networks," Physica A, Vol. 388, No. 24, 2009, pp. 4999-5004. doi:10.1016/j.physa.2009.08.029

[42] F. W. S. Lima and D. Stauffer, "Ising Model Simulation in Directed Lattices and Networks," Physica A, Vol. 359, 2006, pp. 423-429. doi:10.1016/j.physa.2005.05.085

[43] P. R. Campos, V. M. Oliveira and F. G. B. Moreira, "Majority-Vote Model on Small World Network," Physical Review E, Vol. 67, No. 2, 2003, Article ID: 026104.

[44] F. W. S. Lima, "Majority-Vote on Undirected Barabási-
Albert Networks," Communications in Computational Physics, Vol. 2, No. 2, 2007, pp. 358-366.

[45] E. M. S. Luz and F. W. S. Lima, "Majority-Vote on Directed Small-World Networks," International Journal of Modern Physics C, Vol. 18, 2007, p. 1251. doi:10.1142/S0129183107011297

[46] F. W. S. Lima, A. O. Sousa and M. A. Sumuor, "MajorityVote Model on Directed Erdos-Renyi Random Graph," Physica A, Vol. 387-389, 2008, pp. 3503-3511. doi:10.1016/j.physa.2008.01.120

[47] S. Galam, "Sociophysics: A Review of Galam Models," International Journal of General Systems, Vol. 17, No. 2, 1990, pp. 191-209. doi:10.1080/03081079108935145

[48] S. Galam, "Social Paradoxes of Majority Rule Voting and Renormalization Group," Journal of Statistical Physics, Vol. 61, 1990, pp. 943-951. doi:10.1007/BF01027314

[49] L. F. C. Pereira and F. G. B. Moreira, "Majority-Vote Model on Random Graphs," Physical Review E, Vol. 71, No. 1, 2005, Article ID 016123. doi:10.1103/PhysRevE.71.016123

[50] S. Galam, B. Chopard, A. Masselot and M. Droz, "Emergence in Multi-Agent Systems Part II: Axtell, Epstein and Young's Revisited," European Physical Journal B, Vol. 4, No. 4, 1998, pp. 529-531. doi:10.1007/s100510050410

[51] S. Galam, "Collective Beliefs versus Individual Inflexibility: The Unavoidable Biases of a Public Debate," Physica A: Statistical Mechanics and Its Applications, Vol. 390, No. 17, 2005, pp. 3036-3054.

[52] S. Gekle, L. Peliti and S. Galam, "Opinion Dynamics in a Three-Choice System," European Physical Journal B, Vol. 45, No. 4, 2005, pp. 569-575. doi:10.1140/epjb/e2005-00215-3

[53] S. Galam, "Sociophysics: A Review of Galam Models," International Journal of Modern Physics C, Vol. 19, No. 3, 2008, pp. 409-440. doi:10.1142/S0129183108012297

[54] R. Wintrobe and K. G"erxhani, "Tax Evasion and Trust: A Comparative Analysis," Proceedings of the Annual Meeting of the European Public Choice Society, 2004. 